\title{
UTILIZAÇÃO DE AVALIAÇÕES DA APTIDÃO FÍSICA RELACIONADAS À SAÚDE POR PROFESSORES DE UMA CIDADE DA REGIÃO DO PAMPA
}

\author{
Marcelo Clarete Seracini Penner, Universidade Federal do Pampa-UNIPAMPA, Bagé, Rio Grande \\ do Sul - Brasil \\ Antonio Evanhoé Pereira de Souza Sobrinho, Universidade da Região da Campanha - URCAMP, \\ Bagé, Rio Grande do Sul - Brasil \\ Letícia Silveira Cardoso, Universidade Federal do Pampa - UNIPAMPA, Bagé, Rio Grande do Sul \\ - Brasil \\ Marta Regina Cezar Vaz, Universidade Federal do Rio Grande - FURG, Rio Grande, Rio Grande \\ do Sul - Brasil \\ Rodrigo de Azambuja Guterres, Universidade da Região da Campanha - URCAMP, Bagé, Rio \\ Grande do Sul - Brasil
}

\section{RESUMO}

O objetivo do presente estudo foi analisar quais as avaliações da ApFRS são realizadas por professores de educação física no âmbito escolar. Tem caráter exploratório-descritivo, realizado com 18 professores de educação física lotados em 09 escolas de ensino médio de Bagé/RS. Houve menção de 05 valências físicas que são avaliadas com fundamentação científica, realizadas por 12(66,66\%) dos 18(100\%) professores de educação física investigados. Havendo a exclusão de 6(33,33\%) entrevistas por não apresentarem nenhum teste ou avaliação com sustentação teórica. A valência física mais referida foi a da Resistência Aeróbica 08(66,66\%), avaliações de Força e Endurance Muscular com 06(50\%), teste de flexibilidade 04(33,33\%) e avaliação da composição corporal 01(8,32\%). Concluímos que a educação física escolar carece de uma maior preocupação com as avaliações das ApFRS dos alunos, e existe pouca exploração das avaliações no cotidiano escolar.

Palavras-Chave: Saúde do adolescente; Aptidão física; Saúde; Educação física escolar.

\section{USING ASSESSMENTS OF PHYSICAL FITNESS RELATED TO HEALTH BY TEACHERS OF A CITY OF THE REGION OF THE PAMPAS}

\begin{abstract}
The aim of this study was to analyze which ApFRS assessments are conducted by teachers of physical education in schools. Is exploratory-descriptive, conducted with 18 physical education teachers crowded into 09 high schools and Bagé/RS. There was mention of 05 physical valences that are evaluated on scientific grounds, held by $12(66.66 \%)$ of $18(100 \%)$ physical education teachers investigated. Having the exclusion of $6(33.33 \%)$ interviews for not having any test or evaluation with theoretical underpinnings. The more physical valence was referred to the Resistance Aerobics 08 (66.66\%), reviews of Strength and Muscular Endurance with 06 (50\%), flexibility test 04 (33.33\%) and body composition assessment $01(8,32 \%)$. We conclude that the physical education needs of a greater concern with the evaluations of ApFRS students, and there is little exploration of evaluations in everyday school life.
\end{abstract}

Key-Words: Adolescent health; Physical fitness; Health; School physical education. 


\section{USO DE EVALUACIONES DE LA CODICIÓN FÍSICA RELACIONADA COM LA SALUD PARA LOS PROFESSORES DE UNA CIUDAD EN LA REGIÓN PAMPEANA}

\section{RESUMEN}

El objetivo de este estudio fue analizar que ApFRS evaluaciones se llevan a cabo por los profesores de educación física en las escuelas. Es exploratorio-descriptivo, realizado con 18 profesores de educación física hacinados en 09 escuelas secundarias en Bagé/RS. No hubo mención de 05 valencias físicas que se evalúan sobre una base científica, en poder de $12(66,66 \%)$ de los 18 (100\%) profesores de educación física investigados. Tener la exclusión de $6(33.33 \%)$ entrevistas por no tener ninguna prueba o evaluación con fundamentos teóricos. La valencia más físico se refirió a la Resistencia Aeróbica $08(66,66 \%)$, los exámenes de fuerza y resistencia muscular con 06 (50\%), prueba de la flexibilidad $04(33,33 \%)$ y la composición corporal de evaluación 01 (8, 32\%). Llegamos a la conclusión de que las necesidades de educación física de una mayor preocupación por las evaluaciones de los estudiantes ApFRS, y hay poca exploración de las evaluaciones en la vida escolar cotidiana.

Palabra-Clave: Salud de adolescente; Aptitud física; Salud; Educación física en la escuela. 


\section{INTRODUÇÃO}

O papel do professor de educação física na escola vai além da prática esportiva, considerado um formador de opiniões e um propagador de saúde, tendo atribuição de proporcionar a atividade física de forma prazerosa para os escolares e assim contribuir para que os mesmos mantenham esta prática por toda vida.

A execução de avaliações de Aptidão Física Relacionadas à Saúde (ApFRS) nos escolares vai ao encontro da promoção da saúde sendo elas um método confiável para mensuração de valências físicas.

Aptidão Física como é a capacidade de realizar um trabalho muscular de maneira satisfatória. ${ }^{1}$ Existe uma grande a necessidade de avaliar e analisar os componentes motores e antropométricos relacionados à saúde de escolares, pelo fato de diversas pesquisas demonstrarem a relação entre ApFRS e obesidade. ${ }^{2}$

As avaliações da ApFRS se mostram como um meio confiável de se conhecer a forma física de um indivíduo e prevenir doenças através de seus protocolos. ${ }^{2-4}$ Para que seja possível fazer avaliações precisas dos nossos avaliados, é necessário selecionar avaliações que tenham validade, confiança e objetividade, tais conceitos são fundamentais para que a ApFRS seja feita mensurada com confiabilidade. ${ }^{5}$ Mas, no entanto, uma dificuldade encontrada esta presente nos poucos trabalhos com a mesma temática em periódicos especializados, a utilização de avaliações das ApFRS por professores é pouco explorada, sendo encontrado artigos com os resultados dos referidos testes e não sua execução no ambiente escolar.

As avaliações da ApFRS de crianças e adolescentes justificam-se por vários motivos, um deles e, é o fato que diversas doenças crônico-degenerativas possuírem seu período de incubação na infância. ${ }^{6} \mathrm{~A}$ aptidão física é importante por toda vida, no desenvolvimento e na manutenção de uma capacidade funcional e para promover a saúde. ${ }^{7}$

Para avaliarmos a ApFRS de um indivíduo devemos levar em consideração os fatores representados por composição corporal, aptidão cardiorrespiratória, força/endurance muscular, flexibilidade. ${ }^{3-8}$ 
Tais fatores podem ser avaliados e medidos com uma série de testes. Neste sentido, foram criados algumas baterias de avaliações da ApFRS para escolares que servem de referência para os professores de educação física, tais como: Bateria de teste do Projeto Esporte Brasil PROESP-BR; ${ }^{9}$ Baterias de testes de Habilidade Motora geral para escolares de 10 a 18 anos, elaborada pela American Alliance for Health, Physical Education and Recreation and Dance - AAHPERD ${ }^{10}$ Propostas Para Avaliação Física em Escolas, ${ }^{5}$ Bateria do American for Health, Physical Education and Recreation - AHPER; ${ }^{11}$ Bateria do Canadian Association For Health, Physical Education And Recreation - CAHPER; ${ }^{11}$ FITNESSGRAM $;{ }^{6}$ Eurofit; ${ }^{6}$ National Children and Youth Fitness Study-NCYFS $;{ }^{6}$ Phisycal Best. ${ }^{6}$

Tais baterias apresentam uma gana de testes e avaliações da ApFRS, sendo eles utilizados no ambiente escolar divididos em:

Avaliação da composição corporal, sendo ela determinadora de gordura corporal e útil no sentido de estabelecer o peso ideal para saúde, havendo uma grande correlação entre obesidade, cardiopatias, diabetes entre outras. ${ }^{7}$

Aptidão cardiorrespiratória ou capacidade aeróbia nada mais é que a capacidade de realizar exercícios de moderada, média e alta intensidade, de natureza dinâmica, com a utilização de grandes grupos musculares por um tempo prolongado, podendo ser avaliada com varias técnicas ${ }^{7}$. Os testes de aptidão cardiorrespiratória são utilizados como forma de avaliar a capacidade que o corpo tem de atender a demanda de oxigênio através da corrente sanguínea, para manter de forma significativa, os esforços exercidos pelos músculos. ${ }^{6}$

Avaliação de força muscular é basicamente a capacidade de executarmos uma contração ou tensão muscular, para haver movimento ou não movimento, ela pode ser classificada por força estática e força dinâmica, sendo avaliada pelo nível de tensão máxima que pode ser produzido por um grupo muscular. ${ }^{4}$

Avaliação da endurance muscular é uma capacidade de um grupo muscular manter níveis elevados de força por certo período de tempo, desta forma podemos supor que tanto força como endurance muscular tem tarefas similares. ${ }^{4}$ 
A flexibilidade pode ser conceituada como capacidade funcional das articulações, para uma amplitude de movimento articular isolado ou de um grupo de articulações. ${ }^{1-7}$

Com os inúmeros testes e avaliações das ApFRS, a utilização ou não utilização dos mesmos pelos professores de educação física escolar se torne uma preocupação, sendo que eles podem ser mensuradores de possíveis problemas de saúde, tais como a obesidade, cardiopatias dentre outro doenças crônico-não-transmissíveis, tendo isto em vista, o objetivo do presentes estudo foi analisar quais avaliações da ApFRS são realizadas por professores de educação física no âmbito escolar na cidade de Bagé/RS.

\section{METODOLOGIA}

O objeto deste estudo constitui-se das ações dos professores de educação física referente às avaliações da ApFRS em escolares. Tem caráter exploratório-descritivo, amostra constituída por 18 professores lotados em 09 escolas de ensino médio de Bagé/RS, município da Região do Pampa, divididos em 12 do gênero masculino e 6 do gênero feminino, sendo 10 indivíduos da amostra pós-graduados e 8 graduados, e, o tempo de experiência profissional na educação física escolar havia variações entre 3 e 29 anos, com média de 17 anos de experiência. Como critério de exclusão para a pesquisa foi utilizado o não-aceite para a realização da mesma.

A coleta de dados ocorreu com a aprovação de Comitê de Ética em Pesquisa na Área da Saúde da Universidade Federal do Rio Grande, (Processo No 23116.003856/2010-15 e Parecer 109/2010). Como procedimento inicial foi realizado o contato com os responsáveis pelas instituições e solicitação da permissão para desenvolver as etapas da pesquisa, após a anuência dos mesmos, agendaram-se as entrevistas semiestruturadas com os professores de educação física durante o seu turno de trabalho.

Aplicou-se uma análise qualiquantitativa, relacionada à frequência dos resultados obtidos nas entrevistas gravadas, sendo utilizado para a gravação um aparelho gravador SONY modelo ICDPX312 e posteriormente transcritas no programa WORD 2003, com questionamento sobre a referência dos professores de educação física à utilização de testes/avaliações da ApFRS em escolares, afim de mensurar a capacidade de determinadas valências físicas dos escolares do ensino médio, faixa etária correspondente ao intervalo 13 - 18 anos de idade. 
Houve a preocupação em assegurar o anonimato dos sujeitos investigados, a ausência de risco a integridade pessoal e profissional dos trabalhadores e das instituições, conforme Resolução do Conselho Nacional de Saúde nº 196/96. Utilizou-se o Termo de Consentimento Livre e Esclarecido do Participante, apresentando-se os objetivos e finalidades da pesquisa, fortalecendo o direito do participante em obter informações a respeito da pesquisa ou mesmo desistir de participar a qualquer momento do processo.

\section{RESULTADOS E DISCUSSÃO}

A distribuição de frequência aplicada aos dados qualitativos tem a função de consubstanciá-los somente. Deste modo, diferentes testes podem ser referidos por um mesmo professor de educação física, ou seja, não há exclusão de dados.

\section{Avaliações de Aptidão Física relacionadas à Saúde na Educação Física Escolar}

Houve menção de 05 valências físicas que são avaliadas com fundamentação científica, utilizadas por $12(66,6 \%)$ dos 18(100\%) professores de educação física investigados. Havendo a exclusão nos resultados de 6(33,3\%) entrevistas por não apresentarem nenhum teste ou avaliação com sustentação teórica, sendo assim, a composição de $100 \%$ foi constituída por 12 entrevistas, foram descritas nos resultados 6 entrevistas, as entrevistas restantes estavam em concordância com as citadas.

A valência física mais referida foi a da Resistência Aeróbica 08(66,6\%) dos 12(100\%). O teste mais frequentemente referido foi o de Cooper, 06(50\%), também o teste Caminha/Corrida de nove minutos, $02(16,6 \%)$.

Entrevista n6: Nós aqui na escola realizamos o teste de cooper para a ver como esta a capacidade aeróbica dos alunos, fazemos sempre no inicio do ano letivo, nosso espaço aqui na escola é bem grande e como é um teste fácil de ser feito a gente faz um uma semana com todo mundo. E para a força muscular a gente faz o salto vertical que é bem simples também e a gente realiza este teste em dias de chuva dentro da sala de aula.

Entrevista n⿳11: A gente tem só uma quadrinha pequena aqui no colégio e eu coloco os guris para correr numa área demarcada e faço o aquele teste de nove minutos com eles, o problema é quando 
chove que a gente tem que ficar em aula que a quadra fica toda alagada, a gente também faz o de abdominais, aquele de um minuto fazendo abdominais e de avaliação física são só estes dois.

Em consecutivo têm-se avaliações de Força e Endurance Muscular com 06(50\%) referência para cada um deles. O teste de força muscular mais realizado foi o de salto vertical 06(50\%), seguido pelo teste de salto horizontal $02(16,6 \%)$. Enquanto no endurance muscular o teste realizado foi o de resistência abdominal 04(33,33\%).

Entrevista $\mathbf{n}^{0}$ 16: Olha de testes físicos eu ando fazendo aqui o de resistência abdominal e o salto vertical, a parte aeróbia eu avalio conforme as aulas vão passando, vendo a melhora na corridas e nos treinamentos das equipes da escola.

Entrevista $n^{0}$ 18: Na escola temos um cuidado enorme com os alunos a cada semestre fazemos avaliações de quatro valências sendo duas por bimestre iniciamos no primeiro bi com o teste de Cooper e de resistência abdominal, no segundo com o do banco de wells e de salto horizontal e vertical. No segundo semestre fazemos um, re-teste com os alunos, os pais sempre são informados das datas dos testes e nós damos aos pais a opção de olhar os testes dos seus filhos, os pais que não vão nos dias dos teste enviam uma autorização assinada para a realização do testes.

O teste de flexibilidade mais utilizado foi a aferição da envergadura 04(33,3\%) posteriormente o banco de Wells 01(8,3\%), e para a avaliação da composição corporal, o calculo do IMC 01(8,3\%).

Entrevista $\mathbf{n}^{\mathbf{0}}$ 12: Eu trabalho apenas com as meninas aqui na escola dando aula de ginastica, a diretora sempre nos pede para fazer testes nos alunos, dai eu faço o teste de Cooper faço a envergadura e o salto horizontal, dai apenas o de cooper é com a turma mista meninos e meninas.

Entrevista $\mathbf{n}^{0}$ 13: O colégio aqui é bem pequeno, mas a gente faz o que pode, meus alunos fazem a corrida de nove minutos, a gente mede a envergadura e faz o teste dos abdominais. Tudo bem tranquilo a gente vai fazendo durante o ano inteiro os testes, conforme o tempo e o cronograma da coordenadora, em algumas comemorações da escola a gente faz o IMC com os pais, com os alunos a gente faz no inicio do ano para já ir avaliando as condições porque depois já começam com jogos escolares e isto na me da uma direção de quem vai para qual esporte. 
Os professores de educação física têm o esporte como foco principal nas aulas tendo em vista o tecnicismo visando a performance e o rendimento ao invés da atividade física voltada para a saúde ${ }^{12}$. Em estudo realizado em uma cidade do interior do Paraná, escolares não apresentaram níveis satisfatórios de aptidão física relacionada à saúde, revelando um risco aumentado a doenças crônicas não transmissíveis aos escolares, mostrado a importância de tais avaliações. ${ }^{2}$

Estudos epidemiológicos têm mostrado redução de pelo menos 50\% nos índices de mortalidade entre indivíduos com escores elevados de aptidão física comparados com o menos aptos fisicamente, mostrando assim a importância de realizar avalições físicas nos escolares. ${ }^{13}$ Os mesmos autores também descrevem que jovens bem condicionados fisicamente estão associados a menor prevalência de fatores de risco predisponentes às cardiopatias, redução da adiposidade total e abdominal e as melhores condições de saúde óssea e mental, imprescindíveis para os escolares

Avaliar e analisar os componentes motores e antropométricos relacionados à saúde de escolares é essencial, pelo fato de diversas pesquisas demonstrarem a relação entre baixos níveis de aptidão física com certas patologias que podem vir a ser instalados precocemente. ${ }^{2}$

\section{CONCLUSÃO}

Concluímos que a educação física escolar na cidade de Bagé deixa a desejar quanto a aplicação das avaliações das ApFRS em escolares, dos entrevistados 6 docentes não realizam qualquer tipo de avaliação com os escolares, assim sendo excluídos da mostra dos resultados, os 12 docentes restantes revelam utilizar mas com pouca exploração das avaliações no cotidiano escolar.

Com um maior numero de professores de educação física realizando avalições da resistência aeróbia ela mostrasse na opinião dos mesmos, muito importante pela sua eficácia e a facilidade da realização de protocolos de avaliação aeróbia também é um fator relevante para a escolha desde tipo de teste ser realizado.

Os professores também se mostram interessados na aferição da resistência muscular e na endurance muscular, sendo muito utilizado para direcionar a escolha de um esporte para o aluno levando em consideração os resultados de testes como o de salto horizontal, vertical e resistência abdominal. 
A flexibilidade não é tão utilizada, mas os professores tem a consciência de sua importância, a composição corporal, tem grande importância na investigação de possíveis distúrbios metabólicos, e mesmo com esta importância ela é utilizada por uma minoria dos profissionais entrevistados.

A estruturação dos planos pedagógicos das escolas perante a educação física influencia na construção das aulas e das atividades relacionadas a esta disciplina, sendo uma reestruturação pedagógica uma medida plausível para a realização de protocolos completos de avaliações das ApFRS. Outros estudos envolvendo esta temática são relevantes para uma melhor compreensão do planejamento educacional relacionado às aulas de educação física.

\section{REFERÊNCIAS}

${ }^{1} \mathrm{HOBOLD}$, E. Indicadores de aptidão física relacionada à saúde de crianças e adolescentes do município de Marechal Cândida Rondon - Paraná, Brasil. 2003. 96 f. Dissertação (Mestrado em Educação Física) - Universidade Federal de Santa Catarina, Florianópolis, 2003.

${ }^{2}$ FONSECA, H. A. R. et. al. Aptidão física relacionada á saúde de escolares de escola pública em tempo integral. Acta Scientiarum: health sciences, Maringá, n. 2, p. 155-161, 2010.

${ }^{3}$ AMERICAN COLLEGE OF SPORTS MEDICINE (ACMS). Manual do ACMS para avaliação da aptidão física relacionada á saúde. 3. ed. Rio de Janeiro: Guanabara Koogan, 2012.

${ }^{4}$ GUEDES, D. P.; GUEDES, J. E. R. P. Manual prático para avaliação: em educação física. Barueri: Manole, 2006. 484p.

${ }^{5}$ PITANGA, F. J. G. Testes medidas e avaliação: em educação física e esportes. São Paulo: Phorte, 2008. 223p.

${ }^{6}$ PERIN, C. M. R.; CONTE, E. M. T. Estudo de protocolos de avaliação da aptidão física relacionada à saúde em escolares. 2008. Disponível em: http://www.diaadiaeducacao.pr.gov.br/portals/pde/arquivos/866-4.pdf. Acesso em: 28 set. 2012. 
${ }^{7}$ AMERICAN COLLEGE OF SPORTS MEDICINE (ACMS). Aptidão física na criança e no adolescente. Revista Brasileira de Medicina do Esporte, São Paulo, v. 3, n. 2, abr./jun. 1997.

${ }^{8}$ PETROSKI, E. L. et. al. Aptidão física relacionada á saúde em adolescentes brasileiros residentes em áreas de médio/baixo índice de desenvolvimento humano. Revista Salud Pública, v. 13, p. 219228, abr. 2011.

${ }^{9}$ GAYA, A.; SILVA, G. Manual de aplicações de medidas e testes, normas e critérios de avaliação. Porto Alegre: PROESP, jul. 2007.

${ }^{10}$ ROCHA, P. E. C. Medidas e avaliações: em ciência do esporte. Rio de Janeiro: Sprint, 2008. 173p.

${ }^{11}$ NUNES, V. G. S.; CAMPOS, A. L. P. Manual prático para medir e avaliar: em educação física. Pelotas: Ed. da UFPEL, 2010. 253p.

${ }^{12}$ SANTOS, M. A. G. N.; NISTA-PICCOLO, V. L. O esporte e o ensino médio: a visão dos professores de educação física da rede publica. Revista Brasileira de Educação Física e Esporte, São Paulo, v. 25 p. 65-78, jan./mar. 2011.

${ }^{13}$ GUEDES, D. P. et. al. Aptidão física relacionada á saúde de escolares: programa fitnessgram. Revista Brasileira de Medicina do Esporte, São Paulo, v. 18, p. 72-76, mar./abr. 2012. 
Conexões: revista da Faculdade de Educação Física da UNICAMP, Campinas, v. 12, n. 4, p. 41-50, out./dez. 2014. ISSN: 1983-9030 